\section{Analytische Phase}

C. Vidal ${ }^{1}$ und W.-R. Külpmann ${ }^{2}$

${ }^{1}$ Landeskriminalamt Niedersachsen, Dezernat 53 „Chemie“, Hannover, Deutschland

${ }^{2}$ Hannover, Deutschland

Synonym(e) Liquorprotein-Labilitätstest

Beschreibung In der analytischen Phase findet die qualitative oder quantitative Untersuchung der $>$ Analysenprobe statt. Ihr geht voraus die Präanalytik ( $\triangleright$ Präanalytische Phase) und ihr folgt die Postanalytik ( $\triangleright$ Postanalytische Phase; z. B. Befundübermittlung).

\section{Literatur}

Stamm D, Büttner J (1995) Klinisch-chemische Analytik. In: Greiling H, Gressner AM (Hrsg) Lehrbuch der Klinischen Chemie und Pathobiochemie, 3. Aufl. Schattauer Verlag, Stuttgart, S 6-36 